\section{Platelet concentrates transfusion - modern immunological issues}

DOI: $10.1515 / \mathrm{rrlm}-2017-0017$

\section{Dear Editor,}

The first blood transfusion was administered in 1492 to Pope Innocent VIII, but safe transfusions started to be practised after 1900 when Landsteiner discovered the ABO blood system. Platelet transfusions developed later during the 1960s and still represent an immunological challenge due to alloimmunization and developing refractory states. Alloimmunization, the most challenging issue in modern transfusiology, represents development of antibodies as a result of exposure to foreign antigens such as blood cell antigens or plasma proteins and usually appears after blood transfusion or postpartum. (1)

The aim of this paper is to review the main antigens involved in alloimunization, to define platelet transfusion refractoriness, and to present several preventive and therapeutic strategies for alloimunisated patients.

\section{Platelet antigens- clinical implications}

On the platelet surface there are several types of antigens: platelet specific antigens and antigens shared with other cells (red blood cells, HLA antigens). (1)

Platelet specific antigens are located on the glycoproteins of the platelet membrane (GPIIIa, GP Ib, GPIIb). Before 1990, several platelet antigens were described named by the patient in whom the pair antibody was originally found (Zw, Ko, Bak, Yuk). The human platelet anti- gen (HPA) nomenclature system was adopted in $1990(1,2)$ and revised in 2003. (3) To date, 24 HPAs have been defined by immune sera. These 24 antigens were grouped into six biallelic systems (HPA-1, -2, -3, -4, -5, and -15) based on having alloantibodies defining a given antigen. (2)

Platelet specific antigens are targets for antibodies (autoantibodies, alloantibodies, drug-dependent antibodies). Alloantibodies appear following transfusion or postpartum and are involved in refractoriness to platelet transfusions. (1)

Clinical situations involving platelet-specific antigens and antibodies are autoimmune thrombocytopenia and drug-induced immune thrombocytopenia (anti-HPA autoantibodies). Alloantibodies are involved in neonatal alloimune thrombocytopenia (Anti-HPA-1a, AntiHPA-5a, HLA-15a), posttransfusional purpura (Anti-HPA-1a, Anti HPA-3b) or refractoriness state to platelet transfusion (all HPA antigens). $(2,4)$.

Antiplatelet specific antibodies can be detected in the serum/sera of politransfused alloimunized patient in $20-25 \%$ of cases. These patients usually associate posttransfusional anti-HLA antibodies, too. (2)

Platelets bind on their surface some erythrocyte specific antigens (ABO, Lewis, Ii and P), but lack antigens like Rh, Duffy, Kell, Kidd. Some ABO antigens are part of the platelet membrane, others are adsorbed from the plasma. The density of A or B antigens on platelets surface is about $5 \%$ of that on erythrocytes (1), this quantity is enough to produce an immune conflict. Duquesnoy (cited by McCullough), one of the pioneers of platelet transfusion, reported a $23 \%$ reduction in platelet recovery after HLAmatched and ABO-incompatible transfusion. 
In this case recipients' antibodies bind A or B transfused antigens. In clinical practice, ABO matched platelet transfusions are rarely administered only in selected cases (children, refractory patient). ABO alloimunization is considered to have less importance than HLA alloimunization in platelet refractoriness issue. $(1,5)$

HLA antigens are integral to the platelet membrane. Platelet HLA antigens are class I (-A, $-\mathrm{B},-\mathrm{C})$, while class II (DR) is not found on platelets (1) or may be induced on the platelet surface by cytokine stimulation (gamma-interferon). (2) Anti-HLA antibodies appear after an alloimunization process. This phenomenon appears postpartum, after administering non-leukodepleted erythrocytes, platelet concentrate transfusions, or after non HLA matched platelet transfusions (like most platelet concentrate transfusion). After non-leukodepleted transfusions, $80-100 \%$ of the patients may develop HLA antibodies against leucocyte antigens. These Anti-HLA/ antibodies may bind HLA antigens situated on transfused platelets and may determine refractoriness. If the patient is treated with therapeutic regimens containing immunosuppressive drugs, HLA antibodies appear only in $40-70 \%$ of cases. The earliest alloimunisation against HLA antigens appears 10 days post-transfusion, but usually anti-HLA antibodies are produced after 3-4 weeks. Posttransfusional alloimunisation does not correlate with the number or frequency of platelets or red blood cell transfusions, but correlates with the number of transfused leucocytes. Using leukodepleted blood transfusions (leucocytes count lower than $1-5 \times 10^{6}$ per unit) could reduce primary immunization against HLA class I antigens. In a large percentage of alloimmunized patients, anti HLA antibodies disappear with time. $(2,6)$

The clinical consequences of posttransfusional alloimunization and platelet transfusion refractoriness could be very severe. Most of the patients suffer malignant diseases treated with chemotherapy and are severely thrombocytopenic. Platelet transfusions are indicated to prevent or treat bleeding. If one of these patients is alloimmunized and platelet transfusions are ineffective, the patient's life could be threatened.

Management and prevention of alloimunization to HLA class I antigens is a modern research issue in transfusion medicine and immunology.

Prevention of alloimunization could be done by removal of leukocytes from blood components (using special filters or specific apheresis techniques), by irradiation, or by transfusing to one patient platelet concentrates from the same donor. (4)

\section{Lack of response to platelet transfusions}

Alloimunization is not equivalent to refractoriness. One patient may have a poor platelet increment after a single transfusion, but may respond well to subsequent transfusions. As such, a diagnosis of refractoriness to platelet transfusion should be established after two or more ABO-compatible transfusions stored less than 72 hours. If one patient has this profile and expresses antibodies associated with platelet destruction, the process is considered "immune refractory". (2)

In order to define a refractory state to platelet transfusion, there is an equation that reflects the expected increment of platelets after a transfusion (a dose-response effect). For the standard increment one hour after transfusion, the platelet count should increase by approximately 10,000/ $\mathrm{mL}$ when $1 \times 10^{11}$ platelets are transfused into a $70 \mathrm{~kg}$ patients. This means that one unit of whole blood derived platelets produce a platelet count increase of 5,000 to $10,000 / \mathrm{mL}$. A more accurate indicator is one-hour corrected count increment (CCI). This is the most commonly used indicator because it depends on the size of the recipient of the transfusion and the number of platelets transfused. The CCI is calculated as follows: 
CCI $=($ Posttransfusion - Pretransfusion Platelet Count) x Body Surface Area / Number of Platelets Transfused. The ideal CCI is 15,000/ $\mathrm{mL} / 10^{11}$ platelets transfused. After one transfusion, if CCI is less than $5,000 / \mathrm{mL}$, the patient is considered to be refractory. $(4,7)$

Poor response to platelet transfusion may be caused by several immune or non-immune factors related to the patient or to the platelet concentrates. Patient related factors are female gender (related to alloimmunization after pregnancies), splenomegaly, bleeding, infection, disseminated intravascular coagulation, number of transfusion (related to alloimmunization, too), prescribed medication (heparin, amphotericin, antibiotics). Furthermore, many patients have more than one clinical factor that produces refractoriness.

Platelet related factors are: $\mathrm{ABO}$ incompatibility and platelets near the end of their storage period (5 days). Several studied have shown that the optimum period for platelet transfusion is 24-48 hours after collection. (4)

\section{Strategies for managing patients re- fractory to platelet transfusions}

\subsection{Use of ABO compatible platelets}

In transfusion management of platelets refractory patients, the first step is treating any correctable clinical factors such as infections, DIC. After that, the correct dose of platelets should be calculated (1 unit/10 kg of body weight). Fresh $(<$ 72 hours old) ABO-identical platelets should be chosen. A study centered on refractory patients proved that $67 \%$ of patients recovered after $\mathrm{ABO}$ identical platelet transfusion, but only $19 \%$ recovered after ABO-incompatible platelets. Other studies proved a relatively small difference between the survival of all ABO- compatible and incompatible platelets. $(2,8)$

\subsection{Use of HLA compatible platelets from identical siblings or unrelated donors.}

This method is expensive and could provide matched platelets for a limited number of patients (low probability of finding an identical HLA donor). Some authors reported that the average response to HLA partially matched transfusion is similar to that of fully HLA- identical transfusions. (2)

Also, by using the HLA-matched platelet transfusions in refractory patients, the failure rate is about $20-50 \%$ depending on the technique of cross-matching (4)

There are two different techniques to provide to the patients with HLA compatible platelets. Firstly, selecting HLA compatible registered donors, and secondly, selecting HLA matched platelet units using cross-matched tests. $\left(2,4,{ }^{9}\right)$

The techniques suitable for cross-matching are: monoclonal anti IGG assay, platelet migration inhibition assay, platelet immunofluorescence technique. $(2,10)$

HLA-compatible platelet transfusions are indicated to refractory patients, especially to HLA alloimmunized patients. HLA antibodies can be detected using laboratory techniques like immunofluorescence test, ELISA or mixed red cell adherence assay. (2)

\subsection{Use of acid treated platelets}

Citric acid removes HLA class I antigens, but decreases in vivo survival of those platelet concentrates. (2)

\subsection{Other approaches to the refractory patients}

Strategies for platelet refractory patients are plasma exchange (removing platelet antibodies), administration of Cyclosporine as an immunosuppressive drug for reducing the production of antiplatelet alloantibodies or administration of intravenous immunoglobulin (having the same 
mechanism of action as in autoimmune thrombocytopenia). All these methods may have a therapeutic effect, but are not largely used. (4)

Alloimunization to platelet transfusions represents a complication that could be prevented (using leukodepleted blood cell components and adopting a restrictive transfusion policy) rather than treated. The modern approach of these patients assumes an interdisciplinary team (clinician, transfusiologist, laboratory medicine specialist).

\section{Andrada Pârvu', ${ }^{1,2}$ Iulia-Andrea Zsoldos', Anca Bojan ${ }^{1}$}

\section{5. "Iuliu Hatieganu” University of Medicine} and Pharmacy, Cluj-Napoca, Romania;

6. "Prof. dr. Ion Chiricuța" Oncological Institute, Cluj-Napoca, Romania;

*Corresponding author: Andrada Pârvu e-mail: parvuandrada@hotmail.com

Received: 25th February 2017;

Accepted: $9^{\text {th }}$ April 2017;

Published: 18 $8^{\text {th }}$ April 2017.

\section{References}

1. McCullough J. Blood groups. In: McCullough J, editor. Transfusion Medicine. 2nd ed. Philadelphia: Elsevier; 2005. p. 181-219.

2. Mollison PL. Immunology of leucocytes, platelets and plasma components. In: Mollison PL, editor. Blood Transfusions in Clinical Medicine. 11th ed. Malden, Mass: Blackwell Publishing; 2005, p. 546-610.

3. Metcalfe P, Watkins NA, Ouwehand WH, Kaplan C, Newman P, Kekomaki R, et al. Nomenclature of human platelet antigens. Vox Sanguinis. 2003 Oct;85(3):240245. DOI: 10.1046/j.1423-0410.2003.00331.x

4. McCullough J. Clinical uses of blood components. In: McCullough J, editor. Transfusion Medicine. 2nd ed. Philadelphia: Elsevier; 2005. p. 251-307.

5. Valsami S, Dimitroulis D, Gialeraki A, Chimonidou M, Politou M. Current trends in platelet transfusions practice: The role of $\mathrm{ABO}-\mathrm{RhD}$ and human leukocyte antigen incompatibility. Asian J Transfus Sci. 2015 JulDec;9(2):117-23. DOI: 10.4103/0973-6247.162684

6. Network for the Advancement of Patient Blood Management, Haemostasis and Thrombosis. RBC Filtration and Allogeneic Blood Transfusion. Available at: $\quad$ http://www.nataonline.com/np/147/rbc-filtration-and-allogeneic-blood-transfusion [cited on 03.apr.2017]

7. Stanworth SJ, Navarrete C, Estcourt L, Marsh J. Platelet refractoriness - practical approaches and ongoing dilemmas in patient management. Br J Haematol. 2015 Nov;171(3):297-305. DOI: 10.1111/bjh.13597

8. Cowan K. Strategies to reduce inappropriate use of platelet transfusions. Nursing Times [online]. 2017;113(2):18-21.

9. Vassallo RR, Fung M, Rebulla P, Duquesnoy R, Saw $\mathrm{CL}$, Slichter SJ, et al. Utility of cross-matched platelet transfusions in patients with hypoproliferative thrombocytopenia: a systematic review. Transfusion. 2014 Apr;54(4):1180-91. DOI: 10.1111/trf.12395

10. Heikal NM, Smock KJ. Laboratory testing for platelet antibodies. Am J Hematol. 2013 Sep;88(9):818-21. DOI: $10.1002 /$ ajh.23503 